tion of BHMs for climate reconstruction. Dr. Andrew Gelman of Columbia University gave the opening keynote address, and Drs. Martin Tingley of NCAR, Bo Li of Purdue University, Johannes Werner of the University of Giessen, Matthew Schofield of the University of Kentucky, and Naresh Devineni of Columbia University led the workshop with regard to the use and implementation of BHMs for spatially explicit climate reconstruction.

A second important purpose of the workshop was to explore how the more established multivariate regression based methods performed in comparison to BHMs, and to examine the extent to which the traditional methods could offer equally or near-equally valid ways to characterize reconstruction uncertainties in practice (see Fig. 1). This latter goal is important due to the additional complexity and computational expense of BHM approaches, and the more formal and complete treatment of uncertainties afforded by BHMs.

A strong focus was also put on separating model building, per se, from inference of model parameters. It was noted that climate scientists sometimes mix these two concepts, which can result in significant attention being paid to inference issues and comparisons of performance within a closely-related set of models (such as "flavors" of regression, cf., Bürger et al., 2006), rather than to the more general issue of developing conceptually appropriate yet computationally tractable models. In this regard, the key shift in thinking is not to Bayesian methods but to models-which would likely be hierarchical in nature. Inference can then be conducted using a range of tools, but as models become more involved, Bayesian inference strategies may be the (conceptually) simplest option.

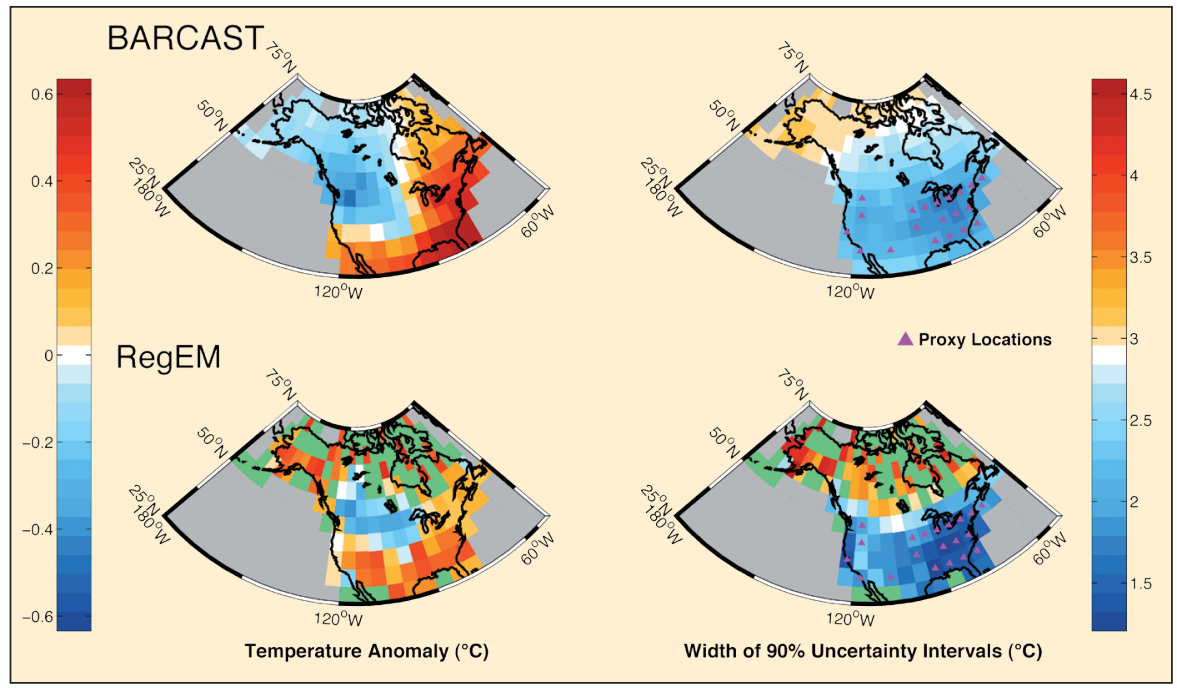

Figure 1: Results of a pseudo-proxy reconstruction experiment comparing Bayesian estimates from BARCAST (upper row; Tingley and Huybers, 2010) with frequentist estimates from RegEM (lower row; Schneider, 2001), an important "state-of-the-art" approach to climate field reconstruction. The left column shows point estimates of the temperature field, while the right column gives the width of $90 \%$ uncertainty estimates and indicates the locations of the pseudo-proxies. Results are for the year 1890 of the "medium" experiment described in Tingley and Huybers (2010). The additional assumptions made by BARCAST allow for spatially complete inference, while RegEM does not provide inference at locations where there are no instrumental observations during the calibration interval (indicated by the green shading)

Several presenters stressed that BHMs are not "one size fits all". A given model, such as BARCAST (Tingley and Huybers, 2010), may be appropriate for inferring a particular target process from a particular data set, in the sense that all diagnostics indicate the modeling assumptions are suitable, the Markov Chain Monte Carlo (MCMC) estimation process converges, and the resulting ensemble of draws has reasonable properties. However, the same model may produce results that are physically unreasonable or otherwise problematic if applied to a different data set, or used to infer a different target process examples of which were presented and discussed in the workshop. Such results can often be interpreted as an indication of model misspecification, and it was stressed that model building is an iterative process. Akin to the residual analysis that follows standard linear regression, BHMs allow for posterior checks of the suitability of the model assumptions for the data under analysis.

Bayesian Hierarchical Modeling is still in its infancy in the context of paleoclimate field reconstructions. A key goal of this workshop was to develop a common language, and to focus on formalizing scientific understanding through collaboration between paleoclimate scientists and statisticians. This first (and hopefully not last) workshop took significant steps towards enabling this necessary collaboration to proceed.

\section{References}

Bürger, G., Fast, I. and Cubasch, U., 2006: Climate reconstruction by regression-32 variations on a theme, Tellus, 58A: 227-235

Schneider, T., 2001: Analysis of Incomplete Climate Data: Estimation of Mean Values and Covariance Matrices and Imputation of Missing Values, Journal of Climate, 14(5): 853-871.

Tingley, M.P. and Huybers, P., 2010: A Bayesian Algorithm for Reconstructing Climate Anomalies in Space and Time, Part I: Development and Applications to Paleoclimate Reconstruction Problems Journal of Climate, 23: 2759-2781.

\title{
Synthesis of transient climate evolution of the last 21 ka (SynTRaCE-21) workshop
}

\section{Mount Hood, Oregon, 10-13 October 2010}

\section{Zhengyu Liu' ${ }^{1}$, B. Otto-Bliesner ${ }^{2}$ and P. Clark ${ }^{3}$ \\ 'Department of Atmospheric and Oceanic Sciences, University of Wisconsin-Madison, USA; zliu3@wisc.edu \\ ${ }^{2}$ Climate and Global Dynamics Division, National Center for Atmospheric Research, Boulder, USA; ${ }^{3}$ Department of Geosciences, Oregon State University, Corvallis, USA.}

Climate reconstructions covering the last 21 ka provide critical observational data for testing state-of-the-art climate models for the simulation of climate evolution and abrupt climate changes. New proxy evidences and modeling activities have led to rapid advances in our understanding of climate change for this period. Therefore, a new PAGES Working Group, SynTraCE-21, was initiated in 2009 to synthesize the transient climate evolution of the last $21 \mathrm{ka}$. The overarching goals of the
Working Group and the associated workshop series are (i) to facilitate an international synthesis effort of proxy climate records to better describe the major features of global climate during the last $21 \mathrm{ka}$, and (ii) to compare these data to transient 


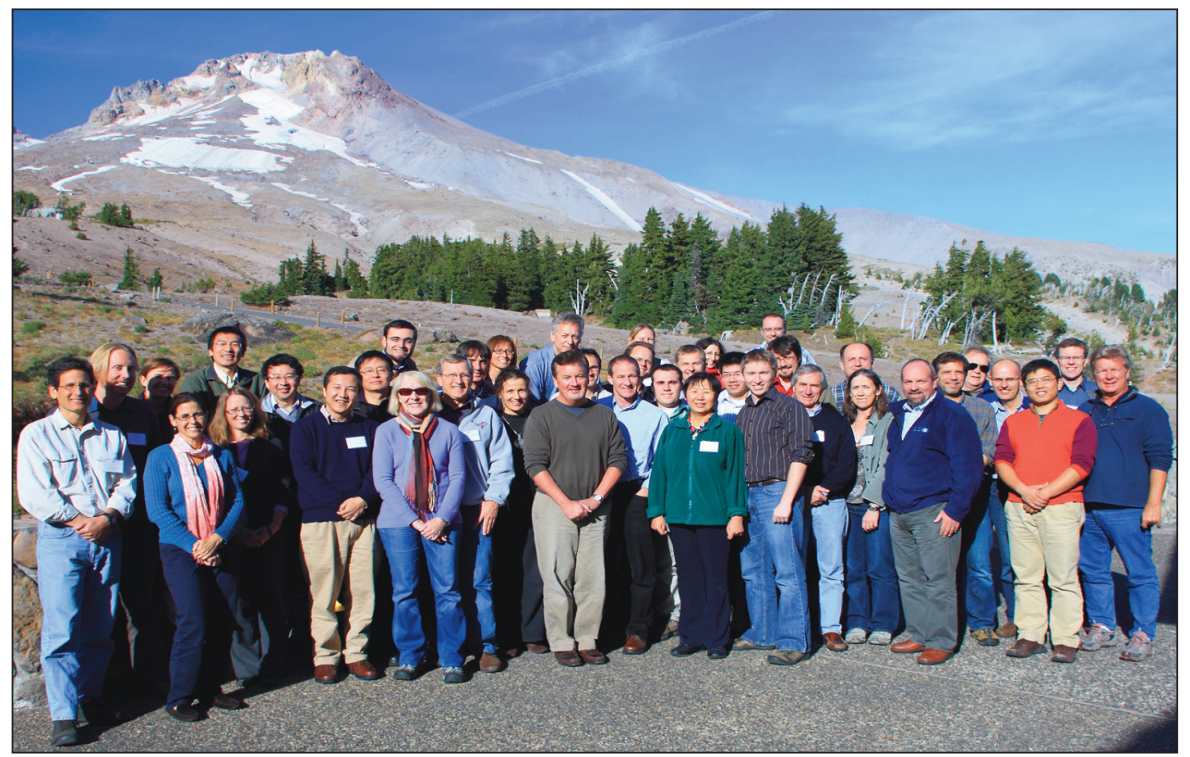

Figure 1: SynTraCE-21 meeting participants in the foreground, Mount Hood in the background.

model simulations. The first international SynTraCE-21k workshop was supported by PAGES, NOAA, and the US Department of Energy, and attracted more than 40 participants from around the world with expertise from terrestrial and marine paleoclimatology to climate modeling.

The first day began with a review of two previously held pilot workshops, which focused on marine and terrestrial records (held in Madison August 2008, and Boulder August 2009). Following this, the focus turned to an update of the meltwater history, which is the most uncertain component of climate forcing in the last deglaciation. The rest of the day was devoted to preliminary model-data comparisons in three models, two coupled general circulation models (CCSM3 and HadCM3) and a climate model of intermediate complexity (ECBilt). These transient simulations mark a new era beyond the "snapshot" studies on "time-slice" climate in paleoclimate model-data comparison because they allow for a direct comparison of time series between the model and data. The studies presented ranged from the evolution of monsoons and global surface climate to regional abyssal circulation variability, showing the great potential of these transient simulations for model-data comparison.

Transient simulations provide an unprecedented opportunity to the paleoclimatology community for modeldata comparison and for improved understanding of climate evolution and abrupt climate change. As a result, it has become critical to develop a major data synthesis to better characterize the global climate variability and to compare with the new generation of transient model simulations.

The second day focused on the terrestrial proxies, with synthesis discussions of lake sediment records, including pollen and charcoal data, ice cores and speleothems. A special session was also arranged to discuss several model-data comparison strategies, including both forward and inverse modeling. The third day was devoted to marine proxies. Reconstructions of the character of the deep and intermediate waters with sedimentary isotopes studies were described. Finally, surface ocean proxies for sea surface temperature and salinity were discussed. Each day ended with an open discussion on the major topics of the day.

The presentations on each major proxy provided a great learning opportunity to all the participants, greatly promoting the interdisciplinary approach towards a multi-proxy data synthesis. To examine model robustness, the meeting participants also recommended coordinated modeling activities among the different research groups. To better assess modelmodel differences and climate sensitivities to external forcing, notably to meltwater forcing, while allowing for flexibility for such long simulations, one strategy proposed was to design common standard sensitivity experiments for different models. Finally, given the large amount of model data, a coordinated model data distribution was also discussed. The workshop participants agreed that the next meeting would be held in the summer of 2012. This workshop will focus on several key topics using an interdisciplinary synthesis approach. Notably, the topics will include the meltwater history and sea level reconstructions for the deglaciation, climate and terrestrial ecosystem in the North American region, and tropical hydrology.

\title{
The $3^{\text {rd }}$ PAGES Past Interglacials workshop
}

\author{
Palisades, New York, USA, 20-22 October 2010
}

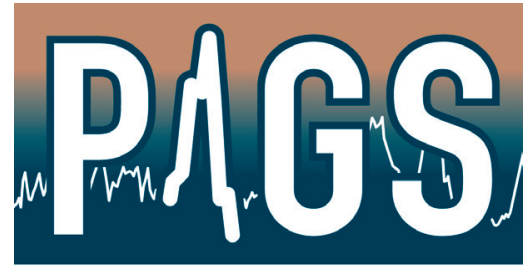

PAST INTERGLACIALS

Jerry F. McManus ${ }^{1}$, D. Raynaud ${ }^{2}$ and P.C. Tzedakis ${ }^{3}$

'Lamont-Doherty Earth Observatory of Columbia University, New York, USA; ${ }^{2}$ Laboratory of Glaciology and Geophysical Environment (LGGE), Grenoble, France; ${ }^{3}$ Department of Geography, University College London, UK; p.c.tzedakis@ucl.ac.uk

Uncertainties related to climate variability in a warming world lend high priority to studies of warm periods in the past. The interglacial intervals of the last 800 ka serve as particularly appropriate targets for such investigations (Tzedakis et al., 2009), as they represent the culmination of warming over a range of partially ice-free global climates. In order to help focus and coordinate international efforts to study these intervals, the PAGES Working Group on Past Interglacials (PIGS) held the third in a series of workshops at the Lamont-Doherty Earth Observatory of Columbia University.
Previous workshops laid out the PIGS themes and addressed intra-interglacial variability and interglacial onset. The meeting in New York brought together 30 scientists representing the marine, terrestrial, ice core and modeling communities to focus on interglacial duration (Fig. 1) and glacial inception. Participants came 